\title{
Suelos potenciales para el cultivo de camu camu (Myrciaria dubia H.B.K. McVaugh) en la provincia de Coronel Portillo, región Ucayali
}

\author{
Study of Potential Soils for the Camu Camu (Myrciaria dubia H.B.K. McVaugh) Farming in the \\ Province of Coronel Portillo, Ucayali Region
}

\author{
Wagner G. Verde Bedoya ${ }^{1}$ y Julio C. Nazario Ríos ${ }^{2 *}$
}

* Autor de correspondencia

\begin{abstract}
Resumen
El objetivo del estudio fue determinar las zonas aptas potenciales para el cultivo de camu camu (Myrciaria dubia H.B.K. McVaugh). Las zonas potenciales fueron determinadas al aplicar siete criterios (geológico, clasificación de suelos, fisiografía, clasificación de capacidad de uso mayor, textura del suelo, distancia del ro y nivel de inundación). También, se elaboró un mapa temático por cada criterio, obteniéndose de la superposición de los mapas las zonas potenciales para el sembrado del cultivo de camu camu. Se encontró que del total de la superficie evaluada, el sector de Masisea posee tierras en condiciones aptas, regulares y no aptas para el sembrado de camu camu en porcentajes de $12,2 \%, 82,2 \%$ y $3,7 \%$ respectivamente. El sector de Mashangay posee tierras en condiciones aptas y regulares para el sembrado de camu camu en porcentajes de 10,7\% y 88,7\% respectivamente. El sector de Yarinacocha posee tierras en condiciones aptas, regulares y no aptas para el sembrío de camu camu en porcentajes de 22,1\%, 43,4\% y 27,9 respectivamente. Por otro lado, el sector de Pucallpillo posee tierras en condiciones aptas, regulares y no aptas para el sembrío de camu camu en porcentajes de $10,54 \%, 60,85$ y $23,3 \%$ respectivamente. Por último, el sector de Utuquinia posee tierras en condiciones aptas, regulares y no aptas para el sembrío de camu camu en porcentajes de $17,0 \%, 23,7 \%$ y 53,11\% respectivamente.
\end{abstract}

Palabras clave: camu camu; cultivos en limpio y permanentes; inundabilidad; terraza baja.

\begin{abstract}
The objective of the study was to determine potential suitable areas for cultivation of camu camu (Myrciaria dubia H.B.K. McVaugh). Potential zones were determined by applying seven criteria (geologic, soil classification, physiography, land capability classification, soil texture, distance of the river and flooding level). Also, a thematic map was elaborated for each criterion, obtaining from the superposition of the maps the potential zones for the cultivation of camu camu. It was found that of the total area evaluated, the Masisea sector owns land in suitable conditions, regular and unsuitable for the planting of camu camu in percentages of $12,2 \%, 82,2 \%$ and 3,7\%, respectively. The Mashangay sector has lands in suitable and regular conditions for the farming of camu camu in percentages of $10,7 \%$ and $88,7 \%$, respectively. The Yarinacocha sector has lands in suitable, regular and not suitable conditions for the farming of camu camu in percentages of $22,1 \%, 43,4 \%$ and $27,9 \%$, respectively. On the other hand, the Pucallpillo sector has lands in suitable, regular and not suitable conditions for the farming of camu camu in percentages of $10,54 \%, 60,85 \%$ and 23,3\%, respectively. Finally, the Utuquinia sector has lands in suitable, regular and not suitable conditions for the farming of camu camu in percentages of $17,0 \%, 23,7 \%$ and $53,11 \%$, respectively.
\end{abstract}

Keywords: Camu camu; permanent and clean farming; floodability; low terraces.

\section{Introducción}

El camu camu (Myrciaria dubia H.B.K. McVaugh), es uno de los cultivos que se encuentra en proceso de expansión debido principalmente a la demanda del mercado y por el contenido de vitamina C (2700 mg de ácido ascórbico/100 $\mathrm{g}$ de pulpa). Por esa razón, con la finalidad de atender el boom comercial suscitado, el Estado del Perú a partir de 1997 impulsó la promoción de plantaciones de camu camu, recomendando su instalación en 10000 hectáreas en los departamentos de Loreto y Ucayali; asimismo, el
Ministerio de Agricultura a través del Decreto Supremo 046-99-AG del 25 de noviembre de 1999, considera al camu camu como un cultivo de interés nacional, y con Resolución Ministerial 21-2000-AG, del 19 de enero del 2000, se aprueba el Programa Nacional de Camu Camu, que tiene como objetivos promover el desarrollo sostenible e integral del camu camu en función del mercado, a fin de mejorar la calidad de vida del poblador amazónico y potenciar las riberas de los ríos, arroyos y lagos en armonía con el medio ambiente. 
El camu camu (Myrciaria dubia H.B.K. McVaugh) crece de manera natural en las orillas de los ríos, cochas y cursos menores de agua en la amazonía. Su distribución natural indica que la mayor concentración de poblaciones y de diversidad se encuentra en la amazonía peruana a lo largo de los ríos Ucayali y Amazonas y sus afluentes, ocupando posiciones fisiográficas de terrazas bajas y en muy bajas concentraciones en la parte de Brasil (Villachica, 1996). Esta demanda creciente del mercado nacional e internacional está generando que el Estado y las empresas nacionales promuevan la expansión de nuevas áreas productivas. Sin embargo, sin tener la información y conocimiento necesario se ha sembrado camu camu (Myrciaria dubia H.B.K. McVaugh) en suelos no adecuados para el desarrollo de este cultivo, por lo que se hace necesario estudiar las características físicas, químicas, y la fertilidad de los suelos donde crecen de manera natural cerca de las orillas de los ríos y su relación con la inundación que estos puedan sufrir durante el año. Es por ello que es importante determinar las zonas potenciales para la expansión de nuevas áreas de cultivo del camu camu. El objetivo general del estudio fue determinar las tierras potenciales para el sembrío del camu camu (Myrciaria dubia H.B.K. McVaugh) en cinco sectores (Masisea, Mashangay, Pucallpillo, Yarinacocha y Utuquinia), en la provincia de Coronel Portillo, región de Ucayali, mientras que los objetivos secundarios fueron: Identificar las unidades geológicas en los cinco (05) sectores de estudio (Masisea, Mashangay, Pucallpillo, Yarinacocha y Utuquinia); Identificar las unidades fisiográficas en los cinco (05) sectores de estudio (Masisea, Mashangay, Pucallpillo, Yarinacocha y Utuquinia); Clasificar los suelos mediante el "Keys to Soil Taxonomy" (USDA SCS 2014) en los cinco (05) sectores de estudio (Masisea, Mashangay, Pucallpillo, Yarinacocha y Utuquinia); Clasificar las tierras de acuerdo con su capacidad de uso mayor en los cinco (05) sectores de estudio (Masisea, Mashangay, Pucallpillo, Yarinacocha y Utuquinia); Determinar la cercanía del río en la relación con los sectores de estudio (Masisea, Mashangay, Pucallpillo, Yarinacocha y Utuquinia); Determinar las zonas inundables en el área de estudio en los cinco (05) sectores de estudio (Masisea, Mashangay, Pucallpillo, Yarinacocha y Utuquinia).

\section{Generalidades del cultivo del camu camu (Myrciaria dubia H.B.K. McVaugh, 1964)}

El camu camu (Myrciaria dubia H.B.K. McVaugh) es un frutal nativo de la amazonía peruana constituyendo su hábitat natural las zonas inundables en la naciente del Amazonas y los estuarios y riberas del Ucayali. Según McVaugh (1968), citado por Villachica (1996), pertenece a la familia botánica Myrtaceae, género Myrciaria. Este género no es muy amplio, habiéndose estudiado poco de la taxonomía del camu camu y se le ha clasificado como Myrciaria dubia (H.B.K) Mc Vaugh. A pesar de ser una especie nativa de las zonas aluviales inundables, el camu camu prospera bien en los suelos con buen drenaje, siempre y cuando exista un buen suministro hídrico. Es así que la planta crece bien en las cercanías de la localidad de Pucallpa, donde la precipitación pluvial es de 1700 $\mathrm{mm} / \mathrm{año}$. En este caso, el mejor desarrollo se obtiene en suelos aluviales inundables periódicamente, luego, en los suelos con drenaje deficiente y por último en los suelos con drenaje normal (Villachica, 1996). En la región de Ucayali la inundación por la creciente del río Ucayali dura tres a cuatro meses (entre enero a abril), las cuales coinciden con la época de fructificación (Sánchez, 2006).

En cuanto al requerimiento edáfico, las tierras aluviales son los sitios ideales para el cultivo del camu camu, por ser una especie que tolera muy bien las inundaciones. Pero no debe generalizarse, pues no todas las tierras aluviales son apropiadas para una producción rentable y duradera (Achung, 1991), debiéndose excluir aquellos suelos muy superficiales, es decir con menos de $50 \mathrm{~cm}$ de profundidad, suelos arenosos, inundados permanentemente, o aquellos con alto riesgo de poder desbarrancar. Los lugares más apropiados están en las restingas o terrazas aluviales medias y altas, con suelos francos (contenidos de arena, limo y arcilla más o menos en la misma proporción), y donde el nivel de agua de las inundaciones regulares no llega a amenazar la integridad de las cosechas (IIAP, 2001),

Las características químicas de los suelos aluviales varían, observándose un $\mathrm{pH}$ extremadamente ácido en rodales naturales, habiéndose encontrado valores de $\mathrm{pH}$ que fluctúan de 3,25 a 4,66. En los orillares de aguas blancas, con plantaciones de camu camu en buen estado, se encontraron valores de $\mathrm{pH}$ de 6,1 a 7,3, clasificados como ligeramente ácidos a neutros. Se infiere que el camu camu desarrolla, adecuadamente, en suelos variados en $\mathrm{pH}$, desde muy ácidos a neutros. En cuanto al contenido de materia orgánica, valores medios entre 2\% y 4\% son considerados adecuados. En los rodales naturales, los valores de materia orgánica son mayores (rango de 3,8 a $12 \%$ ). En estos suelos con niveles bajos de nitrógeno $(0,06 \%)$, se observó desarrollo normal de plantas adultas, sin presentar síntomas por deficiencia. En general, los valores de nitrógeno, en rodajes naturales, son superiores a los encontrados en los orillares de ríos de aguas blancas. Los niveles medios de fósforo (P), superiores a 7 ppm, son favorables para el camu camu. En los rodales naturales se encontraron niveles notablemente altos, entre 17 a 46 ppm de $\mathrm{P}$, mientras que en restinga, los valores son de nivel medio a alto, entre 7 a 21 ppm de P (IIAP 2001).

\section{Ecosistemas recomendables}

El IIAP 2001 describe los ecosistemas inundables de la amazonía, los cuales forman un complejo de opciones que presentan diferencias críticas en relación con niveles de inundación, nivel de sedimentación y erosión lateral. Como se muestra en la Figura 1, la mayor distancia de la plantación al río da lugar a claras desventajas en términos de disponibilidad de nutrientes, presencia de plantas invasoras y organismos perjudiciales, lo que se refleja en mayores costos. 


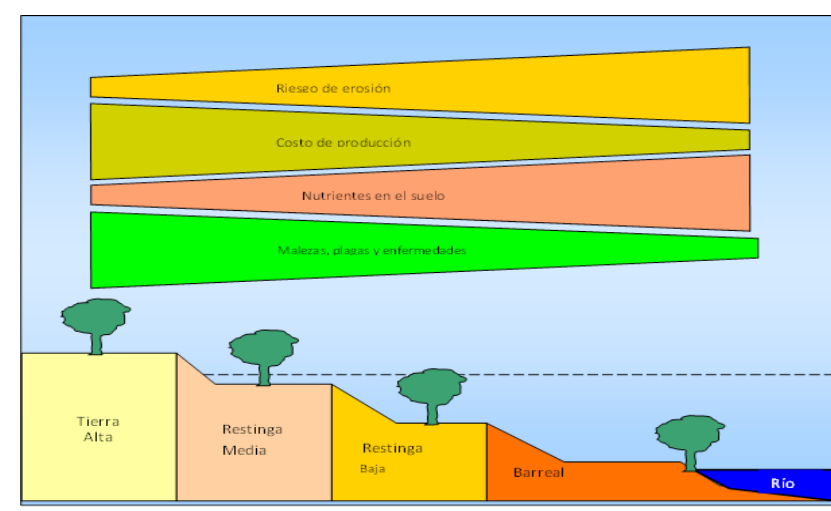

Figura 1. Ecosistemas recomendables

Fuente: IIAP (2001)

La inundación en los suelos aluviales permite un adecuado control de malezas, regulación de poblaciones de insectos y nivel satisfactorio de nutrientes. Las terrazas altas tienen condiciones adecuadas para sostener un sistema como el del camu camu. Sin embargo, la mayor diversidad y abundancia de malezas encarece las labores de mantenimiento, la regulación de insectos se complica por su mayor persistencia y los niveles nutritivos son menores que en los pisos más bajos. Una ventaja que podría tener este piso es su mayor estabilidad en relación con los riesgos de erosión y de inundaciones tempranas. Evidentemente, pequeñas diferencias en altitud ejercen grandes diferencias ecológicas, biológicas y productivas (Peters \& Vasquez, 1986).

\section{Materiales y métodos}

El estudio se localizó en la región de Ucayali, en la provincia de Coronel Portillo y en los distritos de Callería, Masisea, Manantay y Yarinacocha. En estos distritos se escogieron cinco (05) sectores de estudio que son los siguientes: sector Masisea con 6 639,8 ha; sector Mashangay con 1 507,3 ha; sector Pucallpillo con 4 081,3 ha, sector Yarinacocha con 8 350,1 ha; y el sector Utuquinia con 8463,1 ha.

La metodología del estudio siguió una secuencia de cuatro etapas. La primera etapa correspondió a actividades de gabinete (precampo) donde se realizaron la adquisición y procesamiento de imágenes satelitales y la ubicación tentativa de las calicatas y preparación de materiales para el trabajo de campo; la segunda etapa, el trabajo de campo comprende la evaluación de los puntos de muestreo donde se describió la zona circundante al punto de muestreo y la descripción morfológica del perfil registrándose el número y espesor de horizontes, color (en húmedo $\mathrm{y}$ seco), textura al tacto, consistencia (en seco, húmedo $\mathrm{y}$ mojado), estructura (tipo, tamaño y grado), presencia de carbonatos, modificador textural, moteaduras, distribución y tamaño de raíces, reacción del suelo, presencia de películas de arcilla y límite de horizontes (Porta \& López, 2005). En los cinco sectores evaluación se realizaron treinta y cuatro (34) calicatas y se obtuvieron en total ciento tres (103) muestras. La tercera etapa comprendió la fase de laboratorio y la cuarta etapa correspondió a la interpretación del trabajo de campo, resultados de laboratorio y la determinación de las zonas potenciales para el sembrío del camu camu.

Criterios para la determinación de la potencialidad de las tierras para el sembrío del camu camu; se ha aplicado criterios establecidos por el Instituto de Investigación de la Amazonía Peruana (IIAP 2001), como nivel de inundación, posición fisiográfica, distancia mínima $500 \mathrm{~m}$ de la orilla del rio y la textura del suelo. Además, se propuso incluir las características geológicas, que son muy importantes para poder inferir algunas características de formación, como la edad y origen; el criterio pedológico, por estudiar al suelo en su ambiente natural y el criterio de capacidad de uso mayor de la tierra. Para cada criterio se elaboró un mapa con tres niveles de ponderación, apta, regular y no apta. Para obtener la potencialidad de las tierras se ha superpuesto los siete mapas temáticos como geología, suelos, capacidad de uso mayor, fisiografía, textura, clasificación de acuerdo a la distancia del río y nivel de inundación. El mapa final de potencialidad fue obtenido mediante un análisis multivariado, en el cual fueron sumados digitalmente por el SIG los siete mapas. En este procedimiento se utilizó el siguiente algoritmo, modificado de Sánchez et al. (2004).

$P C=\frac{G e(p p)+S u(p p)+F i(p p)+C U M(p p)+I n(p p)+T x(p p)+D r(p p)}{N t v}$

Donde PC representa potencialidad del sembrío de camu camu; pp, peso ponderado de la variable; Ge, Geología; Su, Suelos; Fi, Fisiografía; CUM, capacidad de uso mayor; In, inundabilidad; Tx, textura; Dr, distancia del río y Ntv; número total de variables.

En el Tabla 1 se muestra la información que presentaba cada polígono en el mapa, donde se asignaba un código, y a cada código se le agregaban las variables correspondientes a la temática tratada; $y$, al final, se tenía el producto de haber aplicado la fórmula anterior. Finalmente se estableció un índice de aptitud potencial (PC) de las tierras para el cultivo de camu camu ver Tabla 2 .

Tabla 1. Secuencia de operación de aptitud

\begin{tabular}{ccccccccc}
\hline \multirow{2}{*}{$\begin{array}{c}\text { Código de } \\
\text { polígono }\end{array}$} & $\begin{array}{c}\text { Geología } \\
\text { Ge }\end{array}$ & Suelos & CUM & $\begin{array}{c}\text { Fisiografía } \\
\text { Fi }\end{array}$ & $\begin{array}{c}\text { Inundabilidad } \\
\text { Su }\end{array}$ & $\begin{array}{c}\text { Textura } \\
\text { In }\end{array}$ & Potencial \\
1a & 2 & 2 & 1 & 2 & 2 & 3 & 3 & PC \\
1b & 2 & 2 & 1 & 3 & 2 & 2 & 1 & 2 \\
1c & 1 & 1 & 1 & 2 & 1 & 1 & 3 & 1 \\
\hline
\end{tabular}


Tabla 2. Aptitud potencial para el camu

\begin{tabular}{ccl}
\hline PC & Potencial & \multicolumn{1}{c}{ Descripción } \\
\hline 1 & Apto & $\begin{array}{l}\text { Son tierras adecuadas para el sembrío del camu } \\
\text { camu }\end{array}$ \\
2 & Regular $\begin{array}{l}\text { Son tierras que presentan condiciones regulares } \\
\text { para el sembrío de camu camu. }\end{array}$ \\
3 & No apto $\begin{array}{l}\text { Son tierras que no reúnen condiciones para el } \\
\text { desarrollo de cultivo }\end{array}$ \\
\hline
\end{tabular}

\section{Resultados y discusión}

\section{Sector de Masisea}

El sector en evaluación ocupa una superficie de 6 639,8 ha, donde se describieron la geología, fisiografía, suelos, uso mayor, textura, inundabilidad y las tierras potenciales para el sembrío de camu camu. De acuerdo con el mapa geológico, el área de estudio se encuentra sobre un depósito aluvial de holoceno (Qh-al3) (Rasanen, 1993). La fisiografía se caracteriza por presentar un paisaje de llanura, con un subpaisaje llanura aluvial y dos elementos del paisaje, terraza baja y terraza media. De acuerdo con la clasificación de suelos USDA (2014), se identificaron tres subgrupos: Typic Udifluvents, Mollic Udifluvents y Oxyaquic Udifluvents, todos pertenecen al orden Entisols, que son suelos de reciente formación. En la clasificación de la capacidad de uso mayor de la tierra se identificaron dos subclases: A2si y A3s, ambos pertenecen al grupo cultivos en limpio. La textura del suelo varía de franco limoso a franco arcilloso, y en todos hay predominancia de limo con porcentajes mayores de $40 \%$. En cuanto a la Inundabilidad, el área de estudio presentaba zonas no inundables y zonas inundables. Las zonas inundables se clasificaron en dos rangos: la primera, menor de $1 \mathrm{~m}$; y la segunda, entre 1 a $2 \mathrm{~m}$ sobre el nivel del suelo. De la ponderación de los criterios de evaluación se ha determinado las tierras potenciales (Tabla 3 ).

Tabla 3. Superficie de tierras potenciales en el sector de Masisea

\begin{tabular}{lccc}
\hline Descripción & Potencial & \multicolumn{2}{c}{ Superficie } \\
& PC & ha & $\%$ \\
\hline Aptas & 1 & 815,8 & 12,29 \\
Regular & 2 & 5458,2 & 82,21 \\
No aptas & 3 & 246,0 & 3,71 \\
Otras áreas & & 119,7 & 1,8 \\
\hline Total & & 6639,8 & 100,00 \\
\hline
\end{tabular}

\section{Sector de Mashangay}

Posee una superficie de 1507,3 ha. Geológicamente se encuentra constituido por dos depósitos aluviales: uno del Holoceno (Qh-al3) y el segundo del Pleistoceno que es más antiguo, habiéndose depositado hace 2,5 millones de años aproximadamente (Rasanen, 1993). La fisiografía se caracteriza por presentar un paisaje de llanura, con un subpaisaje llanura aluvial y dos elementos del paisaje terraza baja y terraza media. De acuerdo con la clasificación de suelos USDA (2014), se identificaron tres subgrupos:
Typic Udifluvents y Oxyaquic Udifluvents que pertenecen al orden Entisols y el subgrupo Fluventic Eutrudepts que es un Inceptisols. En la clasificación de la capacidad de uso mayor de la tierra se identificaron tres subclases: A2si, A2s y A3s, todas pertenecen al grupo cultivos en limpio. La textura del suelo varía de franco limoso a arcilloso donde hay predominancia de fracciones de limo mayores de $34 \%$ y de arcilla también mayores de $30 \%$. En cuanto a la Inundabilidad presentan zonas no inundables y zonas inundables. Las zonas inundables se han clasificado en dos rangos: la primera, menor de $1 \mathrm{~m}$; y la segunda, entre 1 a $2 \mathrm{~m}$ sobre el nivel del suelo. De la ponderación de los criterios de evaluación se ha determinado las tierras potenciales (Tabla 4).

Tabla 4. Superficie de tierras potenciales para el sembrío de camu camu en el sector de Mashangay

\begin{tabular}{lccc}
\hline \multirow{2}{*}{ Descripción } & Potencial & \multicolumn{2}{c}{ Superficie } \\
\cline { 3 - 4 } & PC & ha & $\%$ \\
\hline Apto & 1 & 161,4 & 10,7 \\
Regular & 2 & 1336,7 & 88,7 \\
Otras áreas & & 9,2 & 0,6 \\
Total & & 1507,3 & 100,0 \\
\hline
\end{tabular}

\section{Sector de Yarinacocha}

El sector en evaluación ocupa una superficie de 8 350,1 ha, donde se describieron la geología, fisiografía, suelos, uso mayor e inundabilidad y las tierras potenciales para el sembrío de camu camu. De acuerdo con el mapa geológico el área de estudio está constituida por un depósito aluvial (Qh-al3), que corresponde al Cuaternario Holoceno y se halla sobre una llanura inundable (Rasanen, 1993). La fisiografía se caracteriza por presentar un paisaje de llanura, con subpaisaje de llanura aluvial y presenta un elemento de paisaje terraza baja. De acuerdo con la clasificación de suelos USDA (2014), se identificaron dos subgrupos: Oxyaquic Udifluvents y Typic Fluvaquents, ambos pertenecen al orden Entisols y son suelos de reciente formación. En la clasificación de la capacidad de uso mayor de la tierra se identificaron tres grupos: el primero, tierras aptas para cultivos con la subclase A3si; el segundo, tierras aptas para cultivos permanentes con la subclase C3si; y el último, tierras de protección con la unidad Xsi. La textura del suelo varía de franco limoso a arcilloso, y en la mayoría hay predominancia de fracciones de limo con porcentajes mayores de $40 \%$. En cuanto a la Inundabilidad el sector de Yarinacocha solo presentan zonas inundables, las que han sido clasificadas en tres rangos: la primera, menor de $1 \mathrm{~m}$; la segunda, de 1 a $2 \mathrm{~m}$; y la última, mayor de $2 \mathrm{~m}$ sobre el nivel del suelo. De la ponderación de los criterios de evaluación se ha determinado las tierras potenciales ver Tabla 5 .

\section{Sector de Pucallpillo}

Posee una superficie de 4 081,3 ha. Geológicamente se encuentra sobre un depósito aluvial (Qh-al3), que 
correspondiente al Cuaternario del Holoceno y lo constituyen los depósitos fluviales y aluviales (Rasanen, 1993). La fisiografía se caracteriza por presentar un paisaje de llanura, con un subpaisaje llanura aluvial y dos elementos de paisaje terraza baja y terraza media. De acuerdo con la clasificación de suelos USDA (2014), se identificaron dos subgrupos: Typic Udifluvents y Oxyaquic Udifluvents, ambos pertenecen al Orden Entisols y son suelos de reciente formación. En la clasificación de la capacidad de uso mayor de la tierra se identificó un grupo que son las tierras aptas para cultivos en limpio con dos subclases: A3si y A3s. La textura del suelo varía de franco arenoso a arcilloso, y en la mayoría hay predominancia de fracciones de limo con porcentajes mayores de $40 \%$ y de arcillas mayores de $30 \%$. En cuanto a la Inundabilidad presentan zonas no inundables y zonas inundables. Las zonas inundables se han clasificado en tres rangos: la primera, menor de $1 \mathrm{~m}$; la segunda, de $1 \mathrm{a} 2 \mathrm{~m}$; y la última, mayor de $2 \mathrm{~m}$ sobre el nivel del suelo. De la ponderación de los criterios de evaluación se ha determinado las tierras potenciales (Tabla 6).

Tabla 5. Superficie de tierras potenciales en el sector de Yarinacocha

\begin{tabular}{lccc}
\hline \multirow{2}{*}{ Descripción } & \multirow{2}{*}{ Potencial PC } & \multicolumn{2}{c}{ Superficie } \\
\cline { 3 - 4 } & & ha & $\%$ \\
\hline Aptas & 1 & 1846,9 & 22,1 \\
Regular & 2 & 3613,0 & 43,3 \\
No aptas & 3 & 2327,2 & 27,9 \\
Otras áreas & & 562,9 & 6,7 \\
\hline \multicolumn{1}{c}{ Total } & & 8350,1 & 100,0 \\
\hline
\end{tabular}

Tabla 6. Superficie de tierras potenciales para el sembrío de camu camu en el sector de Pucallpillo

\begin{tabular}{lccc}
\hline \multirow{2}{*}{ Descripción } & Potencial & \multicolumn{2}{c}{ Superficie } \\
\cline { 3 - 4 } & PC & ha & $\%$ \\
\hline Apto & 1 & 430,3 & 10,54 \\
Regular & 2 & 2483,8 & 60,86 \\
No apto & 3 & 952,6 & 23,34 \\
Otras áreas & & 214,6 & 5,26 \\
\hline Total & & 4081,3 & 100,0 \\
\hline
\end{tabular}

\section{Sector de Utuquinia}

Posee una superficie de 8 463,1 ha. Geológicamente se encuentra sobre un depósito aluvial del holoceno (Qh-al3) del Cuaternario Holoceno y lo constituyen los depósitos fluviales y aluviales (Rasanen, 1993). La fisiografía se caracteriza por presentar un paisaje de llanura, con un subpaisaje llanura aluvial y un elemento de paisaje terraza baja. De acuerdo con la clasificación de suelos USDA (2014), se identificaron dos subgrupos: Oxyaquic Udifluvents y Aquic Udifluvents, ambos pertenecen al Orden Entisols y son suelos de reciente formación. En la clasificación de la capacidad de uso mayor de la tierra se identificaron dos grupos: el primero son tierras aptas para cultivos en limpio con la subclase A2si; y el segundo son tierras aptas para producción forestal con la subclase F2si. La textura del suelo varía de franco limoso a arcilloso, y en la mayoría hay predominancia de fracciones de limo con porcentajes mayores de $42 \%$ y la fracción arcilla en promedio 34\%. En cuanto a la Inundabilidad el sector de Utuquinia solo presentan zonas inundables, las que han sido clasificadas en tres rangos: la primera, menor de $1 \mathrm{~m}$; la segunda, de 1 a $2 \mathrm{~m}$; y la última, mayor de $2 \mathrm{~m}$ sobre el nivel del suelo. De la ponderación de los criterios de evaluación se ha determinado las tierras potenciales (Tabla 7).

Tabla7. Superficie de tierras potenciales en el sector de Utuquinia

\begin{tabular}{lccc}
\hline \multirow{2}{*}{ Descripción } & Potencial & \multicolumn{2}{c}{ Superficie } \\
\cline { 3 - 4 } & PC & ha & $\%$ \\
\hline Aptas & 1 & 1445,84 & 17,08 \\
Regular & 2 & 2007,71 & 23,72 \\
No aptas & 3 & 4511,72 & 53,31 \\
Otras áreas & & 497,8 & 5,89 \\
Total & & 8463,1 & 100,00 \\
\hline
\end{tabular}

\section{Suelos según su origen}

Todas las estaciones de muestreo se ubicaron sobre una llanura aluvial, en las cuales se ha distinguido los siguientes elementos de paisaje: terraza baja 1 "barrizal", la terraza baja 2 (terraza inundable, donde la inundación es periódica), terraza media plana, terraza media ligeramente ondulada y pantanos, denominando a este último como zonas saturadas por agua gran parte del año. La terraza baja 1 "barrizal" se ha identificado en el sector de Masisea y Yarinacocha, caracterizándose por formarse todos los años, encontrarse muy cerca del curso del río principal o secundario y se inunda generalmente todos los años. La terraza baja 2 se presenta en todo los sectores de estudio, y se caracteriza por inundarse todos los años, donde la altura de inundaciones es muy variable, desde los $10 \mathrm{~cm}$ hasta mayores de $2 \mathrm{~m}$ de altura. La terraza media plana se presenta en los sectores de Masisea, Mashangay y Pucallpillo, caracterizándose por no estar afecto a inundaciones periódicas o prolongadas. La terraza media plana ligeramente ondulada se observa en el sector de Masisea, y corresponde a suelos aluviales que actualmente se encuentran a un nivel de 20 a 25 metros sobre el río Ucayali. Su relieve es ligeramente ondulado. El pantano se ha observado en el sector de Yarinacocha, el cual se caracteriza por encontrarse cubierto con agua gran parte del año, mostrando una geoforma cóncava de succión.

En el área de estudio, los suelos han sido clasificados de acuerdo con la clasificación USDA (2014) encontrándose los siguientes subgrupos:

\section{a. Subgrupo Typic Udifluvents}

Este subgrupo se presenta en tres sectores (Masisea, Mashangay y Pucallpillo), y agrupa a ocho suelos. Se 
distribuye sobre una terraza media plana, que se caracteriza por no sufrir inundaciones en la época de crecidas del río Ucayali. Entre sus características químicas destaca un $\mathrm{pH}$ del suelo que fluctúa entre ligeramente ácido a moderadamente básico, en superficie y profundidad. Vásquez (2000), menciona que el camu camu, en condiciones naturales se desarrolla en suelos con variado $\mathrm{pH}$, desde ácidos hasta de reacción neutra, mientras que, en suelos de altura (Ultisols), el $\mathrm{pH}$ varía entre extremadamente ácido a ligeramente ácido. El contenido de materia orgánica (M.O.) es menor de 3\% disminuyendo con la profundidad, y por consiguiente el contenido de nitrógeno es bajo; en contraste, en los rodales naturales de camu camu los contenidos de M.O. son mayores de 3,8\% (Villachica et al., 1996). La concentración de fósforo disponible es baja (menor de $7 \mathrm{ppm}$ ) en la mayoría de suelos disminuyendo drásticamente con la profundidad; en cambio, el contenido de potasio disponible es medio a alto, disminuyendo también con la profundidad. El contenido de carbonato de calcio es considerado como medio, aumentando con la profundidad, al igual que el calcio intercambiable. Por lo descrito y por las exigencias del cultivo, estos requieren mayores cantidades de materia orgánica y fósforo disponible, por lo cual se considera que la fertilidad edáfica es pobre. Las exigencias nutricionales del cultivo de camu camu pueden ser manejadas con una fertilización química y orgánica, constituyendo la gran desventaja que tiene este suelo para el cultivo la alta incidencia de malezas y plagas, lo cual se traduce en mayor costo de producción.

\section{b. Subgrupo: Mollic Udifluvents}

Este subgrupo se presenta en el sector de Masisea y agrupa dos suelos. Se distribuye sobre posiciones fisiográficas de terraza medias ligeramente onduladas. Entre sus características químicas destaca un $\mathrm{pH}$ del suelo que fluctúa entre ligeramente alcalino a moderadamente básico, en superficie y profundidad, el cual está relacionado con el contenido de carbonatos, el cual puede llegar hasta 3,0\% en los horizontes profundos. El aporte de carbonato de calcio se da por el agua del río, el cual es movilizado en el perfil del suelo por las aguas de infiltración y al llegar a los horizontes inferiores, con menores concentraciones de $\mathrm{CO}_{2}$ y de agua, precipitarán y se acumularán (Lippmann, 1973). Por lo tanto, la dirección de la translocación de carbonatos en los suelos puede ser vertical, que es el resultado de una progresiva infiltración del agua de lluvia o de las inundaciones periódicas, que disuelve los carbonatos en los horizontes superiores y los deposita en los horizontes más profundos donde se produce la precipitación de estas sales, generalmente, a causa de una disminución del contenido en agua del $\mathrm{CO}_{2}$ disuelto. El contenido de materia orgánica (M.O.) es menor de $2,4 \%$ y disminuye con la profundidad, lo que indica que sus contenidos son bajos. La concentración de fósforo disponible es muy variable, desde 13,1 ppm y menos de 0,5. La misma relación se observa con el potasio disponible que fluctúa entre $114 \mathrm{ppm}$ hasta $401 \mathrm{ppm}$. Al igual que el subgrupo anterior, este presenta ciertas deficiencias nutricionales, las cuales podrían ser corregidas con aplicaciones de fertilizantes, su limitación principal es la alta incidencia de malezas y plagas al cultivo, al no tener un control natural que se daría por las inundaciones periódicas que sufren las terrazas bajas.

\section{c. Subgrupo Oxyaquic Udifluvents}

Este subgrupo se presenta en cinco sectores (Masisea, Mashangay, Pucallpillo, Utuquinia y Yarinacocha), siendo este subgrupo el de mayor predominancia. Se distribuye en una fisiografía de terraza baja 1 (barrizal) y terraza baja 2 (terrazas con inundaciones periódicas todos los años en meses de mayor precipitación), y de acuerdo con su posición fisiográfica, las terrazas bajas 2 son las más aptas para el sembrío de camu camu. Entre sus características químicas destaca un $\mathrm{pH}$ del suelo que fluctúa entre fuertemente ácido a moderadamente básico, en superficie y profundidad, el cual está relacionado con el contenido de carbonato de calcio. El contenido de materia orgánica es muy variable, fluctúa desde $0,4 \%$ hasta $4,1 \%$, lo que indica que sus contenidos son bajos a altos, y por consiguiente, el contenido de nitrógeno varía de bajo a alto, considerando la alta tasa de mineralización por ubicarse en una zona tropical. El contenido de M.O. en el perfil del suelo es muy errático porque en algunos hay mayor nivel en la mitad del perfil en relación al primer horizonte, y en otros perfiles el contenido de M.O. es mayor en el primer horizonte y disminuye con la profundidad. El fósforo disponible presenta contenidos bajos a medios, relacionándose los bajos contenidos con una inundación menor de $1 \mathrm{~m}$ de altura, y los contenidos medios con una inundación mayor de $1 \mathrm{~m}$ de altura. En cuanto el potasio disponible, los contenidos son bajos a medios y no se observa relación con la inundación.

\section{d. Subgrupo: Aquic Udifluvents}

Este subgrupo se observa en el sector de Utuquinia y se distribuye en una fisiografía de terraza baja 2, donde las inundaciones se producen todos los años durante la máxima creciente del río Ucayali, y de acuerdo con su posición fisiográfica, estas tierras serían aptas para el sembrío de camu camu, pero desde el punto de vista de inundabilidad no lo son, por presentar una altura de agua mayor de $2 \mathrm{~m}$. Entre sus características químicas presenta un $\mathrm{pH}$ del suelo que fluctúa entre fuertemente ácido a neutro. El contenido de materia orgánica (M.O.) es muy alto en superficie $(7,4 \%)$ y disminuye con la profundidad; por consiguiente, el contenido de nitrógeno es alto, considerando la alta tasa de mineralización. En cuanto al fósforo y potasio disponible, estos son bajos. Las condiciones de inundabilidad mayor de $2 \mathrm{~m}$ serían su principal limitación para desarrollar el cultivo de camu camu sobre estos suelos. 


\section{e. Subgrupo Typic Fluvaquents}

Este subgrupo se observa en el sector de Yarinacocha y se distribuye en una fisiografía de pantanos los cuales, generalmente, permanecen inundados en gran parte del año. Las propiedades físicas del suelo se caracterizan por una textura arcillosa, y las características químicas indican que el pH es moderadamente ácido, el contenido de carbonatos es nulo. El contenido de materia orgánica es clasificado como medio, así como el fósforo, y bajo en potasio disponible.

\section{f. Subgrupo Fluventic Eutrudepts}

Este subgrupo pertenece al orden Inceptisols y se presenta en el sector de Mashangay (Suelo Santo Domingo). Se distribuye en una fisiografía de terraza media plana, que se caracteriza por no presentar inundaciones en las épocas de mayor precipitación. Sus propiedades físicas del suelo se caracterizan por una textura arcillosa, y presencia de horizonte "Bw" con desarrollo de estructura en bloques subangulares. Las características químicas indican que el $\mathrm{pH}$ del suelo es fuertemente ácido, la salinidad es nula, así como el contenido de carbonatos. El contenido de materia orgánica es clasificado como bajo, mientras que los niveles de fósforo disponible y potasio disponible son medios.

\section{Tierras Potenciales}

Tierras No aptas, en esta categoría no es posible la instalación del cultivo de camu camu, porque no reúnen condiciones ecológicas ni técnicas. Ecológicas, debido a que la instalación del cultivo involucra el retiro de especies naturales y modificación de ecosistemas naturales y técnica, se refiere a actividades que no se podrían realizar por las condiciones del medio, como podrían ser los barrizales o zonas muy cercanas al río, que corren el riesgo de ser erosionados y perderse la plantación. Agrupan a los subgrupos Typic Fluvaquents y Aquic Udifluvents.

Tierras con Aptitud regular; son aquellas tierras que después de haber sido evaluadas presentan la aptitud de regular, porque uno o más de dos criterios de evaluación se encuentran limitando su potencialidad. Esta limitación se manifiesta en la producción del cultivo y agrupa a los suelos incluidos en los subgrupos Fluventic Eutrudepts,

\section{Typic Udifluvents y Mollic Udifluvents.}

Tierras aptas, son aquellas tierras en la cuales todos los criterios evaluados son los más adecuados para la instalación y el desarrollo del cultivo. Estos cumplen criterios, como desarrollarse sobre materiales geológicos adecuados, la capacidad de uso mayor requerida para el camu camu, la textura del suelo, la inundabilidad que estos presentan, así como, la distancia adecuada del río, condiciones apropiadas para el cultivo y agrupa al suelo del subgrupo Oxyaquic Udifluvents, excepto los que se encuentran en la terraza baja 1 (barrizal).

\section{Conclusiones}

Sector de Masisea: posee como tierras aptas para el sembrío de camu camu 815,8 ha que representan el 12,29\%; las tierras en condición regular ocupan una superficie de 5458,2 ha y representan el $82,2 \%$; y finalmente, las tierras no aptas ocupan una superficie de 246,0 ha, que representan el 3,71\% del sector en evaluación.

Sector de Mashangay: posee como tierras aptas para el sembrío de camu camu 161,4 ha que representan el 10,7\%; las tierras en condición regular ocupan una superficie de 1 336,7 ha, que representan el $88,7 \%$. No se ha identificado tierras no aptas en este sector.

Sector de Yarinacocha: posee como tierras potenciales para la instalación del cultivo de camu camu, 1846,9 ha aptas que representan el 22,12\%; las tierras con condición regular ocupan una superficie de 3613,0 ha que representan el 43,3\%; y las tierras no aptas ocupan una superficie de 2 327,2 ha, representando el 27,9\% del área evaluada.

Sector de Pucallpillo; posee como tierras potenciales para la instalación del cultivo de camu camu, 430,3 ha que representan el 10,54\%; las tierras con condición regular ocupan una superficie de 2438,8 ha que representan el $69,86 \%$; y las tierras no aptas ocupan una superficie de 952,6 ha que representan el 23,34\% del área evaluada.

Sector de Utuquinia; posee como tierras potenciales para la instalación del cultivo de camu camu, 1445,8 ha que representan el 17,08\%; las tierras en condición regular ocupan una superficie de 2007,71 ha, que representan el $23,72 \%$; y las tierras no aptas ocupan una superficie de 4 511,72 ha representado el 53,31\% del área evaluada.

\section{Literatura citada}

Achung, F. R. 1991. Los suelos de la región del Amazonas según unidades fisiográficas. Folia Amazónica, Instituto de Investigación de la Amazonia Peruana. ISSN 1018 5674.

Decreto Supremo 046-99-AG. El Peruano, Lima, Perú, 25 de noviembre de 1999.

Instituto de Investigación de la Amazonia Peruana (IIAP) 2001. Sistema de producción de camu camu en restinga, pp. 143

Lippmann, F. 1973. Sedimentary carbonate minerals. Springer-Verlag (Berlín), pp. 228

Peters, C.H. \& Vásquez, A. 1986. Estudios ecológicos de camu camu Myrciaria dubia. I. Producción de frutos en poblaciones naturales. En: Acta Amazónica 16-17 (Número único). Brasil, pp. 161-174.

Porta, J.C. \& Lopez, A.R. 2005. Agenda de campo de suelos. Información de suelos para la agricultura y el medio ambiente. Univesitat de Lleida. Ediciones Mundi Prensa. pp 541.

Rasanen, M. 1993. La Geohistoria y Geología de la amazonía peruana. En: Amazonía peruana, vegetación húmeda en el llano subandino. R. Kalliola, M. Puhakka 
y W. Danjoy (eds), pp. 43-67. PAUT y ONERN.

Sánchez, R.; Urrego, L.F.; Mayorga, R. y Vargas, G. 2004. Modelo para el pronóstico de la amenaza por deslizamientos en tiempo real. En Simposio latinoamericano de control de erosión. Colombia. Recuperado de: www.ideam.gov.co.

Sánchez, E. 2006. Fenología del cultivo de camu camu (Myrciaria dubia H.B.K. McVaugh, 1964) en Pucallpa, Perú. En: Investigación universitaria 2(2), Pucallpa.

United States Departament Of Agriculture [USDA]. 2014. Soil Conservation Service. 2010. Keys to soil taxonomy. Soil Surver. Div. Staff. Eleventh Edition.

Vásquez, A. 2000. El camu camu: cultivo, manejo e investigación. Edit. Universal S.R.L. Iquitos-Perú, pp. 218

Villachica, H. 1996. El cultivo del camu camu (Myrciaria dubia H.B.K. McVaugh) en la amazonía peruana. TCA. Secretaría Pro Tempore. Pucallpa-Perú, pp. 96.

Villachica, H.; Carvalho, C.; Müller, h. et al. 1996. Frutales y hortalizas promisorios de la Amazonía. FAO-Tratado de Cooperación Amazónica. (En Prensa). 